\title{
Improvement of Oxygen Transfer Capacity by Formation of Crystal Defect on Red-Mud
}

\author{
Byung Chan Kwon', Misook Kang², Tae Jin Lee ${ }^{1 *}$, No-Kuk Park ${ }^{*}$, Jeom-In Baek ${ }^{3}$, \\ Ui-Sik Kim ${ }^{3}$, Ho-Jung Ryu ${ }^{4}$ \\ ${ }^{1}$ School of Chemical Engineering, Yeungnam University \\ 280 Daehak-ro, Gyeongsan, Korea \\ bckwon79@ynu.ac.kr; tjlee@ynu.ac.kr; nokukpark@ynu.ac.kr \\ ${ }^{2}$ Department of Chemistry, Yeungnam University \\ 280 Daehak-ro, Gyeonsan, Korea \\ mskang@ynu.ac.kr \\ ${ }^{3}$ Korea Electric Power Corporation \\ 105 Munji-ro, Yuseong-gu, Daejeon, Korea \\ jibaek@kepco.co.kr; uisik.kim@kepco.co.kr \\ ${ }^{4}$ Korea Institute of Energy Research \\ 152 Gajeong-ro, Yuseong-gu, Daejeon, Korea \\ hjryu @kier.re.kr
}

\section{Extended Abstract}

Chemical looping combustion is a new concept of combustion technology that facilitates the separation of carbon dioxide because it generates heat energy by combusting hydrogen, syngas, and natural gas using lattice oxygen of metal oxide. In this technique, the metal oxide is used as an oxygen carrier to supply oxygen and transfers oxygen by redox reaction behavior, which is oxidized in an air reactor and reduced in a fuel reactor. In the chemical looping combustion process, the metal oxide particle used as an oxygen carrier circulates through two reactors and the lattice oxygen is consumed by a gassolid reaction in a fluidized bed reactor. Therefore, the lattice oxygen must be transported at a high reaction rate. It must be also prepared in the form of particles with a high surface area. Iron oxide is a relatively inexpensive oxygen carrier, and many researchers have studied oxygen transport properties. In this study, the major component of red mud by-produced after eluting aluminum from bauxite in Bayer process was $\mathrm{Fe}_{2} \mathrm{O}_{3}$, which can be used as an oxygen carrier. However, red mud is not suitable for using oxygen in the crystal lattice due to its low surface area. In this study, the red-mud sample was sulfidated at high temperature to improve the lattice oxygen mobility by form lattice defect in the iron oxide crystals. In order to form crystal defects on the red mud, iron oxide was converted to iron sulfide with hydrogen sulfide, and then was re-oxidized by air to remove sulfur components. In these processes, it was possible to generate defects in the crystal structure. The surface area of the defective red mud increased from about $25.9 \mathrm{~m}^{2} / \mathrm{g}$ to $122.1 \mathrm{~m}^{2} / \mathrm{g}$, and the pore volume increased from $0.1714 \mathrm{cc} / \mathrm{g}$ to $0.2803 \mathrm{cc} / \mathrm{g}$. Also, the formation of crystal defects increased the oxygen transfer capacity of red mud from $1.75 \%$ to $2.18 \%$ at $15 \mathrm{vol} . \%$ hydrogen condition. This result means that the amount of oxygen transported during the reduction process was enhanced by about 1.25 times.

\section{References}

[1] B. Wang, R. Yan, H. Zhao, Y. Zheng, Z. Liu and C. Zheng, "Investigation of Chemical Looping Combustion of Coal with $\mathrm{CuFe}_{2} \mathrm{O}_{3}$ Oxygen Carier," Energy Fuels, vol. 25, pp. 3344-3354, 2011. 